Sociologie et sociétés

\title{
Liste des personnes ayant préparé des évaluations de manuscrits pour Sociologie et Sociétés en 1988-1989 et
} 1989-1990

\section{List of Manuscript Evaluators for Sociologie et soci $t$ 1988-1989 and 1989-1990}

Volume 22, numéro 2, automne 1990

Catholicisme et société contemporaine

URI : https://id.erudit.org/iderudit/001447ar

DOI : https://doi.org/10.7202/001447ar

Aller au sommaire du numéro

Éditeur(s)

Les Presses de l'Université de Montréal

ISSN

0038-030X (imprimé)

1492-1375 (numérique)

Découvrir la revue

Citer ce document

(1990). Liste des personnes ayant préparé des évaluations de manuscrits pour Sociologie et Sociétés en 1988-1989 et 1989-1990. Sociologie et sociétés, 22(2),

223-226. https://doi.org/10.7202/001447ar d'utilisation que vous pouvez consulter en ligne. 


\section{Liste des personnes ayant préparé des évaluations de manuscrits pour Sociologie et Sociétés}

La qualité d'une revue scientifique est un long processus collectif de travail auquel sont associés de nombreux collègues de la communauté scientifique nationale et internationale. Plusieurs fois par année, la Direction de Sociologie et sociétés sollicite la collaboration de collègues ainsi invités à garantir et à assurer la plus grande qualité possible à notre revue. En publiant la liste de celles et de ceux qui nous ont apporté leur aide au cours des années 1988-89 et 1989-90 par leurs commentaires portant sur des manuscrits soumis à leur attention, nous voulons souligner le travail de ces artisans de la qualité de notre revue. Nous leur exprimons ici notre reconnaissance et nos remerciements.

L. M.

1988-1989:

M. Pierre W. BÉLANGER, Faculté des sciences de l'éducation, Université Laval

M. Jean-Guy BELLEY, Faculté de droit, Université Laval

M. Paul BERNARD, Département de sociologie, Université de Montréal

M. Léon BERNIER, Institut québécois de recherche sur la culture, Montréal

Mme Marie-Andrée BERTRAND, École de criminologie, Université de Montréal

Mme Marion BLUTE, Department of Sociology, University of Toronto

Mme Françoise BOUDREAU, Department of Sociology and Anthropology, University of Guelph

M. Donald H. CLAIRMONT, Department of Sociology and Social Anthropology, Dalhousie University

M. François COLBERT, Service de l'enseignement du marketing, École des hautes études commerciales, Université de Montréal

M. Jacques COMMAILLE, Centre de recherche interdisciplinaire de Vaucresson, France 
M. André-Pierre CONTANDRIOPOULOS, Groupe de recherche interdisciplinaire en santé, Faculté de médecine, Université de Montréal

M. Pierre DANDURAND, Département de sociologie, Université de Montréal

M. Jacques DOFNY, Département de sociologie, Université de Montréal

M. Andrew EFFRAT, Faculty of Education, York University

M. Hubert GUINDON, Department of Sociology and Anthropology, Concordia University

M. Jacques T. GODBOUT, Institut national de la recherche scientifique, Montréal

M. Pierre J. HAMEL, Institut national de la recherche scientifique, Montréal

M. Wsevolod ISAJIW, Department of Sociology, University of Toronto

M. Frank E. JONES, Department of Sociology, McMaster University

Mme Danielle JUTEAU, Département de sociologie, Université de Montréal

M. Jacques LAMONTAGNE, Faculté des sciences de l'éducation, Université de Montréal

M. Jean LAPOINTE, Département de sociologie, Université d'Ottawa

Mme Nicole LAURIN, Département de sociologie, Université de Montréal

M. Jacques LAZURE, Département de sociologie, Université du Québec à Montréal

M. Guy LECAVALIER, Department of Sociology and Anthropology, Concordia University

M. Camille LEGENDRE, Département de sociologie, Université de Montréal

M. Joseph L. LENNARDS, Department of Sociology, Glendon College, York University

M. Claude LESSARD, Section d'éducation comparée et fondements de l'éducation, Faculté des sciences de l'éducation, Université de Montréal

M. Camilles LIMOGES, Centre de recherche en évaluation sociale des technologies et Département d'histoire, Université du Québec à Montréal

Mme Patricia MARCHAK, Department of Anthropology and Sociology, University of British Columbia

M. Leon MAYHEW, Department of Letters and Sciences, University of California

M. Peter MEIKSINS, Department of Sociology, State University of New York

M. Albert J. MILLS, Faculty of Administrative Studies, Athabasca University

M. André PETITAT, Département de sociologie, Université du Québec à Montréal

M. Marcel RAFIE, Département de sociologie, Université du Québec à Montréal

M. William Joseph REEVES, Department of Sociology, University of Calgary

M. Marc RENAUD, Département de sociologie et GRASP/sst, Université de Montréal

M. Pierre SAINT-ARNAUD, Département de sociologie, Université Laval

M. Alfred SCHWARZ, Département de sociologie, Université Laval

Mme Peta E. TANCRED, Department of Sociology, McMaster University

M. Richard E. TREMBLAY, École de psycho-éducation, Université de Montréal

M. Claude TROTTIER, Département d'administration et politiques scolaires, Université Laval

M. George WEISZ, Department of Humanities and Social Studies in Medicine, McGill University

M. Irving ZEITLIN, Department of Sociology, University of Toronto

1989-1990:

M. Marc ANGENOT, Département de langue et littérature française, Université McGill

M. Pierre BEAUCAGE, Département d'anthropologie, Université de Montréal

M. Paul BERNARD, Département de sociologie, Université de Montréal 
M. Jacques BERNIER, Département d'histoire, Université Laval

M. Léon BERNIER, Institut québécois de recherche sur la culture, Montréal

Mme Marie-Andrée BERTRAND, Département de criminologie, Université de Montréal

M. Stephen BORNSTEIN, Department of Political Science, McGill University

M. Pierre BOUCHARD, Office de la langue française, Montréal

M. Gilles BOURQUE, Département de sociologie, Université du Québec à Montréal

Mme Nicole BOUSQUET, Département de sociologie, Université Laval

Mme Bettina BRADBURY, Département d'histoire, Université de Montréal

M. Jacques BRAZEAU, Département de sociologie, Université de Montréal

M. John DRYSDALE, Department of Sociology and Anthropology, Concordia University

Mme Bonnie CAMPBELL, Département de science politique, Université du Québec à Montréal

M. Claude-Yves CHARRON, Département des communications, Université du Québec à Montréal

Mme Francine COUTURE, Département d'histoire de l'art, Université du Québec à Montréal

Mme Diana CRANE, Department of Sociology, University of Pennsylvania

M. Carl CUNEO, Department of Sociology, McMaster University

M. Ronald D'COSTA, Faculté des sciences sociales, Université d'Ottawa

M. Raymundo DE ANDRADE, Département de sociologie, Université d'Ottawa

M. Christian DESSUREAULT, Département d'histoire, Université de Montréal

M. Alfred DUBUC, Département d'histoire, Université du Québec à Montréal

M. Gilles DUSSAULT, Département d'administration de la santé, Université de Montréal

M. Jean-Marie FECTEAU, Département d'histoire, Université du Québec à Montréal

M. Hervé GAUTHIER, Bureau de la statistique du Québec, Gouvernement du Québec

M. Charles G. GILLESPIE, Department of Political Science, University of WisconsinMadison

M. Cary HECTOR, Département de science politique, Université du Québec à Montréal Mme Marianne KEMPENEERS, Département de sociologie, Université de Montréal

M. Graham KNIGHT, Department of Sociology, McMaster University

M. Jean-Michel LABATUT, Département de sociologie, Université d'Ottawa

M. Jean-Guy LACROIX, Département de sociologie, Université du Québec à Montréal

M. André LAFRANCE, Département de communication, Université de Montréal

Mme Lise LAMARCHE, Département d'histoire de l'art, Université de Montréal

M. Simon LANGLOIS, Institut québécois de recherche sur la culture et Département de sociologie, Université Laval

Mme Jeanne KIRK-LAUX, Département de science politique, Université d'Ottawa

M. Jocelyn LÉTOURNEAU, Département d'histoire, Université Laval

M. André LUX, Département de sociologie, Université Laval

M. Alex MACLEOD, Département de science politique, Université du Québec à Montréal

M. Gilles MARCOTTE, Département d'études françaises, Université de Montréal

M. Christopher McALL, Département de sociologie, Université de Montréal

M. Louis MICHEL, Département d'histoire, Université de Montréal

M. Roland OUELLET, Département d'administration et politique scolaires, Université Laval

M. Gilles PAQUET, Faculté d'administration, Université d'Ottawa

M. Jacques PELLETIER, Département d'études littéraires, Université du Québec à Montréal 
M. André TURMEL, Département de sociologie, Université Laval

M. Pierre SAINT-ARNAUD, Département de sociologie, Université Laval

M. Daniel SALÉE, Department of Political Science, Concordia University

M. Jules SAVARIA, Agence canadienne de développement international, Hull

M. Roger I. SIMON, The Ontario Institute for Studies in Education, Toronto

M. Michael USEEM, Center for Applied Social Science, Boston University

M. André VACHET, Département de science politique, Université d'Ottawa

Mme Louise VANDELAC, Département de sociologie, Université du Québec à Montréal

Mme Denise VEILLETTE, Département de sociologie, Université Laval

Mme Deena WEINSTEIN Department of Sociology, DePaul University, Chicago

M. George WEISZ, Department of Humanities and Social Studies in Medicine, McGill University

M. Jacques ZYLBERBERG, Département de science politique, Université Laval

M. Jean-Pierre WALLOT, Archives nationales du Canada, Ottawa 